\title{
Plasma S100B is not a useful biomarker for tumor burden in neurofibromatosis
}

\section{Citation}

Smith, Miriam J., Sonia Esparza, Vanessa L. Merker, Alona Muzikansky, Miriam A. Bredella, Gordon J. Harris, Ara Kassarjian, et al. 2013. "Plasma S100? Is Not a Useful Biomarker for Tumor Burden in Neurofibromatosis." Clinical Biochemistry 46 (7-8) (May): 698-700. doi:10.1016/ j.clinbiochem.2012.12.007.

\section{Published Version}

doi:10.1016/j.clinbiochem.2012.12.007

\section{Permanent link}

http://nrs.harvard.edu/urn-3:HUL.InstRepos:33788500

\section{Terms of Use}

This article was downloaded from Harvard University's DASH repository, and is made available under the terms and conditions applicable to Other Posted Material, as set forth at http:// nrs.harvard.edu/urn-3:HUL.InstRepos:dash.current.terms-of-use\#LAA

\section{Share Your Story}

The Harvard community has made this article openly available.

Please share how this access benefits you. Submit a story.

\section{Accessibility}




\section{Plasma $\mathrm{S100 \beta}$ is not a useful biomarker for tumor burden in neurofibromatosis}

Miriam J. Smith ${ }^{\mathrm{a}, 1}$, Sonia Esparza ${ }^{\mathrm{a}, \mathrm{b}}$, Vanessa Merker ${ }^{\mathrm{a}, \mathrm{b}}$, Alona Muzikansky ${ }^{\mathrm{c}}$, Miriam A. Bredella $^{\mathrm{d}}$, Gordon J. Harris ${ }^{\mathrm{d}}$; Ara Kassarjian"; Wenli Cai ${ }^{\mathrm{d}}$, James A. Walker ${ }^{\mathrm{a}, \mathrm{f}}$, Victor F. Mautner $^{\mathrm{g}}$, and Scott R. Plotkin ${ }^{\mathrm{a}, \mathrm{b}}$

${ }^{a}$ Department of Neurology, Massachusetts General Hospital, Boston, MA;

${ }^{\mathrm{b}}$ Cancer Center, Massachusetts General Hospital, Boston, MA; ${ }^{\mathrm{c}}$ Biostatistics Center, Massachusetts General Hospital, Biostatistics Center, Boston, MA; ${ }^{\mathrm{d} D e p a r t m e n t ~ o f ~}$ Radiology, Massachusetts General Hospital, Boston, MA; ${ }^{\mathrm{e}}$ Department of Radiology, Corades, S.L., Majadahonda, Spain; ${ }^{\mathrm{f}}$ Center for Human Genetic Research, Massachusetts General Hospital, Boston, MA; ' Department of Neurology, University Hospital, Hamburg, Germany

${ }^{1}$ Present address: Department of Genetic Medicine, St. Mary’s Hospital, Manchester Academic Health Sciences Centre, Manchester, UK

Address correspondence and reprint requests to:

Scott Plotkin, MD, PhD

Massachusetts General Hospital, Yawkey 9E

55 Fruit Street

Boston, MA 02114

Phone: 617-643-1938, Fax: 617-643-2591

Email: splotkin@partners.org 


\begin{abstract}
Objectives: Neurofibromatosis 1 (NF1), NF2, and schwannomatosis are characterized by a predisposition to develop multiple neurofibromas and schwannomas. Currently, there is no blood test to estimate tumor burden in patients with these disorders. We explored whether S100 $\beta$ would act as a biomarker of tumor burden in NF since S100 $\beta$ is a classic immunohistochemical marker of astrocytes, oligodendrocytes and Schwann cells and a small study showed S100 $\beta$ concentrations correlate with the volume of vestibular schwannomas.
\end{abstract}

Design and Methods: We calculated whole-body tumor burden in subjects with NF1, NF2, and schwannomatosis using whole-body MRI (WBMRI) and measured the concentration of S100 $\beta$ in plasma using ELISA. We used chi-square tests and Spearman rank correlations to test the relationship between S100 $\beta$ levels and whole-body tumor burden.

Results: 127 consecutive patients were enrolled in the study (69 NF1 patients, 28 NF2 patients, and 30 schwannomatosis patients). The median age was 40 years, $43 \%$ were male, median whole-body tumor volume was $26.9 \mathrm{ml}$. There was no relationship between the presence of internal tumors and the presence of detectable S100 $\beta$ in blood for the overall group or for individual diagnoses $(p>0.05$ by chi-square for all comparisons). Similarly, there was no correlation between whole-body tumor volume and $\mathrm{S} 100 \beta$ concentration for the overall group or for individual diagnoses $(p>0.05$ by Spearman for all comparisons). 
Conclusions: Plasma S100 $\beta$ is not a useful biomarker for tumor burden in the neurofibromatoses. Further work is needed to identify a reliable biomarker of tumor burden in NF patients.

Keywords: S100B, neurofibroma, schwannoma, biomarker, neurofibromatosis 


\section{Introduction}

The neurofibromatoses are a group of disorders characterized by a genetic predisposition to multiple nerve sheath tumors. The hallmark tumors in neurofibromatosis include neurofibromas in neurofibromatosis 1 (NF1) patients and schwannomas in NF2 and schwannomatosis patients. Currently, there is no non-invasive way to estimate tumor burden in these diseases. Biomarkers currently exist for other tumors, including prostate cancer (prostate specific antigen, PSA), ovarian cancer (CA125), and germ cell tumors (alphafetoprotein, AFP). A biomarker for nerve sheath tumors could help clinical management of these diseases by screening symptomatic patients for an estimation of internal tumor burden.

The S100 family of calcium-binding proteins is predominantly expressed by astrocytes and Schwann cells and S100 $\beta$ is a classic immunohistochemical marker for these cells. S100ß-secreting cells give rise to classic features of the neurofibromatoses including neurofibromas and schwannomas (Schwann cells) and gliomas (glial cells). The S100 $\beta$ subunit has been studied as a biomarker for many disorders, including stroke,

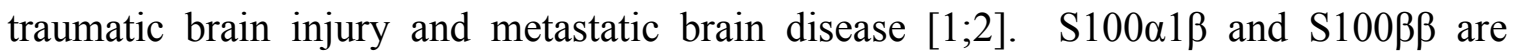
present in malignant tissues, such as melanoma, and have also been found in glioma, thyroid cell carcinoma, and renal cell carcinoma. Determination of S100 $\beta$ in serum has been shown to be clinically useful for prognosis and treatment monitoring of patients diagnosed with stage 3 malignant melanoma [3]. Furthermore, in one study, concentrations of S100 $\beta$ correlated with the volume of vestibular schwannomas [4], which are the hallmark tumor of neurofibromatosis 2. To date, the usefulness of S100 $\beta$ 
levels in estimating internal neurofibroma and schwannoma tumor burden in NF has not been tested.

\section{Material and methods}

\subsection{Patients and imaging}

Samples used in this study were collected from a subset of patients enrolled in a prospective multicenter study of whole-body MRI scanning of subjects with NF1, NF2, and schwannomatosis. Whole-body MRI scans were collected as described previously [5]. In brief, subjects were imaged from head to ankles in the supine position using a 1.5 Tesla magnet, integrated body coil, and no intravenous contrast. Five acquisitions were used to image the entire body with a fat suppressed fluid sensitive STIR sequence. The five images were subsequently fused into a single whole body DICOM image. Noncentral nervous system tumors were identified by a board-certified radiologist and total tumor volume was calculated using semi-automated analysis.

\subsection{Enzyme linked immunosorbent assays}

Blood samples were collected in EDTA-tubes and spun down at 5000x for 10 minutes at $4^{\circ} \mathrm{C}$. Plasma was collected and immediately frozen at $-80^{\circ} \mathrm{C}$. A sandwich model enzyme linked immunosorbent assay (ELISA) (Alpco Diagnostics Ltd, Salem, $\mathrm{NH}$, Catalog number, 48-S1BHU-E01) was used to measure levels of S100 $\beta$ in blood plasma samples. The measurable range of the assay was $98-6300 \mathrm{pg} / \mathrm{ml}$ and the normal physiological serum concentration is $<200 \mathrm{pg} / \mathrm{ml}$ in adults [6]. 


\subsection{Statistics}

Descriptive statistics were calculated for whole-body tumor volume and S100ß concentration for each disease group. Presence or absence of internal tumors and detectable S100ß levels were compared using Chi-square test for categorical variables. The relationship between whole body tumor burden and S100 $\beta$ was analyzed using Spearman coefficients. Statistical analysis was performed using SAS Software (SAS Institute Inc., Cary, NC) and p-values less than 0.05 were considered statistically significant. The study was approved by the institutional review board at Massachusetts General Hospital (Partners Human Research Committee), which waived the requirement for informed consent for this secondary use of samples.

\section{Results}

We analyzed blood samples from 127 consecutive patients enrolled in the research study at Massachusetts General Hospital. This included 69 patients with NF1, 28 patients with NF2, and 30 patients with schwannomatosis. The whole body tumor volume and concentrations of S100 $\beta$ for each group is shown in Table 1. There was no relationship between the presence of internal neurofibromas or schwannomas and the presence of detectable $\mathrm{S} 100 \beta$ in plasma for the overall group or for individual diagnoses by chi-square ( $p>0.05$ for all comparisons). Similarly, there was no correlation between whole body tumor burden and S100 $\beta$ concentration for the overall group or for individual diagnoses (Fig. 1) ( $\mathrm{p}>0.05$ for all comparisons).

\section{Discussion}

NF1, NF2, and schwannomatosis are characterized by phenotypic variability in the number and size of internal nerve sheath tumors both within families and between 
families [7;8] MRI is the gold standard for identifying internal tumors but imaging the entire body is not practical or cost-efficient in the majority of clinical centers. An inexpensive and low-cost laboratory test would be helpful to screen patients for internal nerve sheath tumors. Previous studies suggest that concentrations of S100ß may correlate with the size of vestibular schwannoma, which is the hallmark tumor of NF2 [4]. For this reason, we decided to study whether this marker would correlate with overall tumor burden in patients with a genetic predisposition to nerve sheath tumors (i.e., NF1, NF2, and schwannomatosis).

Our analysis revealed a wide range of $\mathrm{S} 100 \beta$ concentrations among patients. No detectable amounts of S100 $\beta$ were found in $75 \%$ of NF2 patients, $52 \%$ of NF1 patients, and $37 \%$ of schwannomatosis patients. Furthermore, there was no statistically significant correlation between S100 $\beta$ concentration and whole-body tumor burden. Based on this data, we hypothesize that neurofibromas and schwannomas do not release S100 $\beta$ directly into the circulation and that there is an alternative reason for the elevated concentrations seen previously with vestibular schwannomas [4].

The limitations of this study include modest patient numbers within each diagnosis group. Limited ability of WBMRI to image the internal auditory canal prevented us from confirming any relationship between vestibular schwannoma size and S100 $\beta$ levels in NF2 patients. In addition, the WBMRI imaging protocol used does not account for the volume of cutaneous nerve sheath tumors in NF1 and NF2 patients, which may alter $\mathrm{S} 100 \beta$ expression.

To date, no reliable circulating biomarkers have been validated for nerve sheath tumors although adrenomedullin, MIA, and SOX9 have been suggested for future 
development [9-11]. Proteomic analysis of plasma samples from NF and non-NF patients may help identify candidate proteins that correlate with tumor burden.

\section{Conclusion}

Our study does not support the use of plasma $\mathrm{S} 100 \beta$ as a biomarker for tumor burden in neurofibromatosis. Further work is needed to identify a reliable biomarker to screen NF patients for internal tumors without MRI scan. 
Acknowledgements: Support for this project was provided by DOD NF050202 (SRP) and by the Center for Neurofibromatosis and Allied Disorders. The funders had no role in the study design; in the collection, analysis and interpretation of data; in the writing of the report; or in the decision to submit the article for publication. 


\section{References}

[1] M.A. Vogelbaum, T. Masaryk, P. Mazzone, T. Mekhail, V. Fazio, S. McCartney, et al., S100beta as a predictor of brain metastases: brain versus cerebrovascular damage, Cancer 104 (2005), 817-24.

[2] B.J. Blyth, A. Farhavar, C. Gee, B. Hawthorn, H. He, A. Nayak, et al., Validation of serum markers for blood-brain barrier disruption in traumatic brain injury, J. Neurotrauma 26 (2009), 1497-507.

[3] S. Kruijff, E. Bastiaannet, A.C. Kobold, R.J. van Ginkel, A.J. Suurmeijer, H.J. Hoekstra, S-100B concentrations predict disease-free survival in stage III melanoma patients, Ann. Surg. Oncol. 16 (2009), 3455-62.

[4] A.A. Kanner, N. Marchi, V. Fazio, M.R. Mayberg, M.T. Koltz, V. Siomin, et al., Serum S100beta: a noninvasive marker of blood-brain barrier function and brain lesions, Cancer 97 (2003), 2806-13.

[5] S.R. Plotkin, M.A. Bredella, W. Cai, A. Kassarjian, G.J. Harris, S. Esparza, et al., Quantitative assessment of whole-body tumor burden in adult patients with neurofibromatosis, PLoS. One. 7 (2012), e35711.

[6] L.V. Portela, A.B. Tort, D.V. Schaf, L. Ribeiro, D.B. Nora, R. Walz, et al., The serum S100B concentration is age dependent, Clin. Chem. 48 (2002), 950-2.

[7] J.M. Friedman, Neurofibromatosis 1: clinical manifestations and diagnostic criteria, J. Child Neurol. 17 (2002), 548-54.

[8] M.E. Baser, L. Kuramoto, R. Woods, H. Joe, J.M. Friedman, A.J. Wallace, et al., The location of constitutional neurofibromatosis 2 (NF2) splice site mutations is associated with the severity of NF2, J Med Genet. 42 (2005), 540-6.

[9] T.R. Hummel, W.J. Jessen, S.J. Miller, L. Kluwe, V.F. Mautner, M.R. Wallace, et al., Gene expression analysis identifies potential biomarkers of neurofibromatosis type 1 including adrenomedullin, Clin. Cancer Res. 16 (2010), 5048-57.

[10] S.J. Miller, W.J. Jessen, T. Mehta, A. Hardiman, E. Sites, S. Kaiser, et al., Integrative genomic analyses of neurofibromatosis tumours identify SOX9 as a biomarker and survival gene, EMBO Mol. Med. 1 (2009), 236-48.

[11] M. Kolanczyk, V. Mautner, N. Kossler, R. Nguyen, J. Kuhnisch, T. Zemojtel, et al., MIA is a potential biomarker for tumour load in neurofibromatosis type 1, BMC. Med. 9 (2011), 82. 
Figure 1. Relationship between whole-body tumor characteristics and $\mathrm{S} 100 \beta$ concentration in plasma for 127 patients with NF1, NF2, and schwannomatosis.
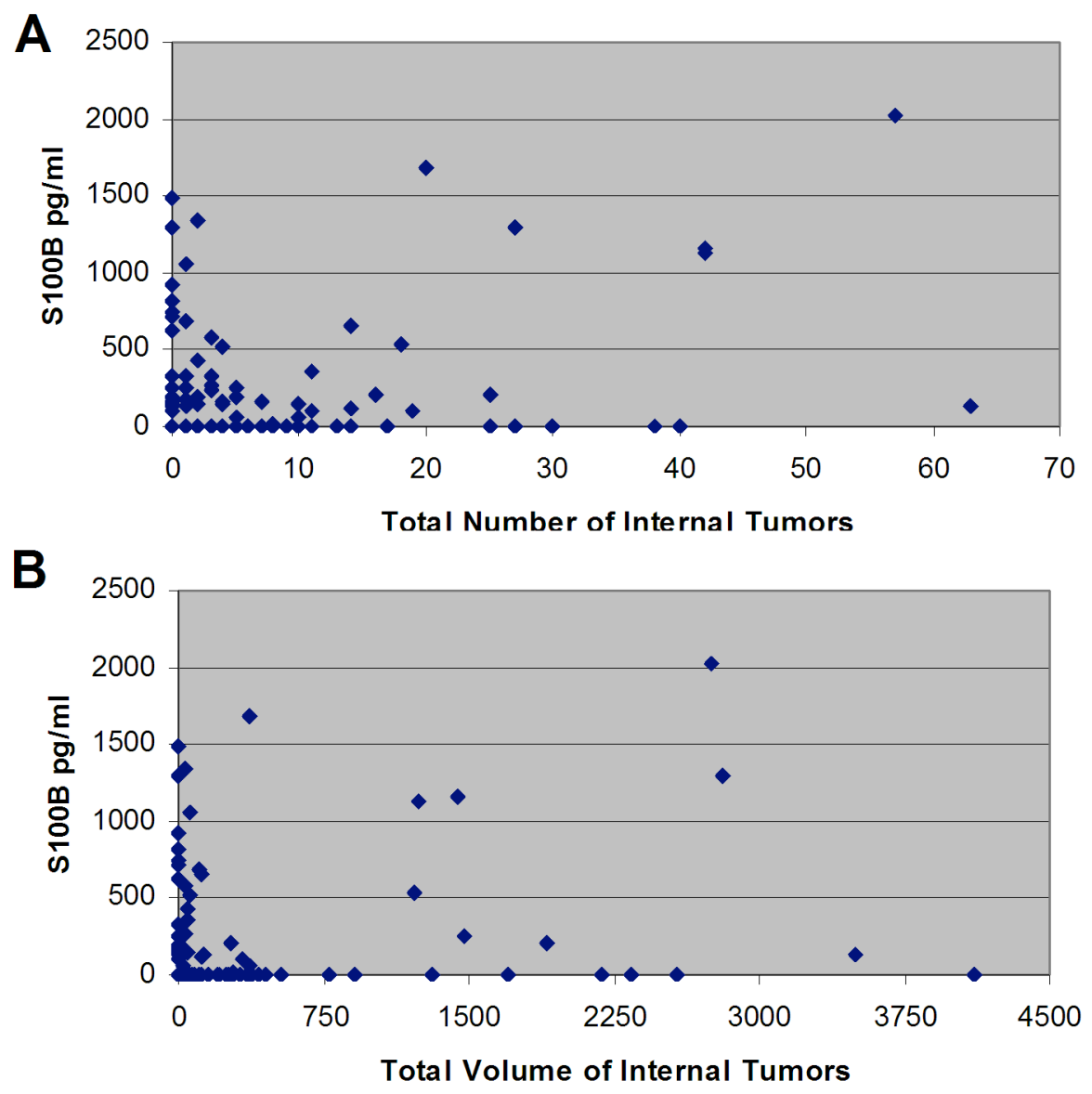

Panel A: Relationship between whole-body tumor number and S100ß concentration.

Panel B: Relationship between whole-body tumor number and S100 $\beta$ concentration, displayed without an extreme outlier of subject with $9,106 \mathrm{ml}$ of tumor and S100ß level of $165 \mathrm{pg} / \mathrm{ml}$. 